PROCEEDINGS OF THE

AMERICAN MATHEMATICAL SOCIETY

Volume 132, Number 10, Pages 3075-3082

S 0002-9939(04)07483-0

Article electronically published on May 12, 2004

\title{
ON THE ASSOCIATED FAMILY OF DELAUNAY SURFACES
}

\author{
M. KILIAN
}

(Communicated by Jon G. Wolfson)

\begin{abstract}
We use the generalised Weierstraß representation of Dorfmeister, Pedit and $\mathrm{Wu}$ to obtain the associated family of Delaunay surfaces and derive a formula for the neck size of the surface in terms of the entries of the holomorphic potential.
\end{abstract}

\section{INTRODUCTION}

The Gauss map of a constant mean curvature (CMC) surface is a harmonic map 11] and Uhlenbeck 15] has shown that such harmonic maps can be obtained as projections of horizontal holomorphic maps from the universal cover of the surface into a certain loop group. There is a Weierstraß type representation for this procedure, commonly referred to as the DPW method, due to Dorfmeister, Pedit and $\mathrm{Wu}$ [4. The construction involves solving a linear differential system with values in a loop group and then Iwasawa decomposing the solution to obtain the extended unitary frame of the Gauss map.

The DPW method is analogous to the Weierstraß representation for minimal surfaces insomuch as input data in the form of meromorphic functions on a Riemann surface generate a conformal CMC immersion of the universal cover. If the underlying Riemann surface is not simply connected, it is necessary to investigate various monodromies and solve period problems in order that one member of the resulting associated family is invariant under deck transformations. Recently, progress has been made in constructing CMC immersions of $n$-punctured spheres with Delaunay ends in the DPW approach [7], [8] and [13].

Since Delaunay surfaces are ubiquitous in the study of CMC surfaces with embedded ends [9], we feel the necessity to give a self-contained account of Delaunay surfaces in the DPW framework. Here we thus describe the construction of the associated family of Delaunay surfaces using holomorphic data on the twice-punctured Riemann sphere.

This paper grew out of a part of the author's Ph.D. thesis [6], and it is a pleasure to herewith express my gratitude to my adviser Franz Pedit as well as to Nicholas Schmitt for numerous useful discussions.

Received by the editors March 4, 2003.

2000 Mathematics Subject Classification. Primary 53A10.

Key words and phrases. Delaunay surfaces, DPW method. 


\section{LOOP GROUPS}

We adopt the notation $\operatorname{diag}[u, v]=\left(\begin{array}{ll}u & 0 \\ 0 & v\end{array}\right)$, off $[u, v]=\left(\begin{array}{ll}0 & u \\ v & 0\end{array}\right)$ and begin by collecting some well-known results on loop groups.

For real $r \in(0,1]$ denote the analytic maps of $C_{r}=\{\lambda \in \mathbb{C}:|\lambda|=r\}$ with values in $\mathrm{SL}(2, \mathbb{C})$ by $\Lambda_{r} \mathrm{SL}(2, \mathbb{C})=\mathcal{O}\left(C_{r}, \mathrm{SL}(2, \mathbb{C})\right)$. We have an involution on maps $C_{r} \rightarrow \mathfrak{g l}(2, \mathbb{C})$ given by

$$
\boldsymbol{\sigma}: g(\lambda) \mapsto \sigma g(-\lambda) \sigma^{-1} \text { with } \sigma=\operatorname{diag}[1,-1],
$$

and we denote the twisted $r$-Loop group of $\operatorname{SL}(2, \mathbb{C})$ by

$$
\Lambda_{r} \mathrm{SL}(2, \mathbb{C})_{\sigma}=\left\{g \in \Lambda_{r} \mathrm{SL}(2, \mathbb{C}): \sigma g=g\right\} .
$$

The Lie algebras of these groups, denoted by $\Lambda_{r} \mathfrak{s l}(2, \mathbb{C})_{\sigma}$, consist of analytic maps $g: C_{r} \rightarrow \mathfrak{s l}(2, \mathbb{C})$ that satisfy $\boldsymbol{\sigma} g=g$. We will use the following subgroups of $\Lambda_{r} \mathrm{SL}(2, \mathbb{C})_{\sigma}:$ Let $K=\{\operatorname{diag}[a, 1 / a]: a>0\} \subset \mathrm{SL}(2, \mathbb{R})$, let $I_{r}=\{\lambda \in \mathbb{C}:|\lambda|<r\}$, and denote

$$
\Lambda_{r}^{+} \mathrm{SL}(2, \mathbb{C})_{\sigma}=\left\{g \in \Lambda_{r} \mathrm{SL}(2, \mathbb{C})_{\sigma} \cap \mathcal{O}\left(I_{r}, \mathrm{SL}(2, \mathbb{C})\right): g(0) \in K\right\} .
$$

Let $A_{r}=\{\lambda \in \mathbb{C}: r<|\lambda|<1 / r\}$, and by an abuse of notation set

$$
\Lambda_{r} \mathrm{SU}(2)_{\sigma}=\left\{g \in \Lambda_{r} \mathrm{SL}(2, \mathbb{C})_{\sigma} \cap \mathcal{O}\left(A_{r}, \mathrm{SL}(2, \mathbb{C})\right):\left.g\right|_{S^{1}} \in \mathrm{SU}(2)\right\} .
$$

For $r=1$ we will omit the subscript. Corresponding to these subgroups, we analogously define Lie subalgebras of $\Lambda_{r} \mathfrak{s l}(2, \mathbb{C})_{\sigma}$. Of fundamental importance is a certain Loop group factorisation, the Iwasawa decomposition. We cite McIntosh [10, where it is proven that the multiplication

$$
\Lambda_{r} \mathrm{SU}(2)_{\sigma} \times \Lambda_{r}^{+} \mathrm{SL}(2, \mathbb{C})_{\sigma} \rightarrow \Lambda_{r} \mathrm{SL}(2, \mathbb{C})_{\sigma}
$$

is a diffeomorphism onto. The associated splitting $g=F B$ of $g \in \Lambda_{r} \mathrm{SL}(2, \mathbb{C})_{\sigma}$ with $F \in \Lambda_{r} \mathrm{SU}(2)_{\sigma}$ and $B \in \Lambda_{r}^{+} \mathrm{SL}(2, \mathbb{C})_{\sigma}$ will be called Iwasawa decomposition. The condition $B(0) \in K$ ensures that the factorisation is unique and

$$
\Lambda_{r} \mathrm{SU}(2)_{\sigma} \cap \Lambda_{r}^{+} \mathrm{SL}(2, \mathbb{C})_{\sigma}=\{\operatorname{Id}\} .
$$

\section{DPW METHOD}

Let $\Omega(M)$ denote the holomorphic 1 -forms on a Riemann surface $M$, and define

$$
\Lambda_{-1}^{\infty} \mathfrak{s l}(2, \mathbb{C})_{\sigma}=\left\{\xi \in \mathcal{O}\left(\mathbb{C}^{*}, \mathfrak{s l}(2, \mathbb{C})_{\sigma}\right): \lambda \xi \in \mathcal{O}(\mathbb{C}, \mathfrak{s l}(2, \mathbb{C}))\right\} .
$$

CMC surfaces come in $S^{1}$ families, the associated family. The DPW method [4] constructs conformal CMC immersions of the universal cover $\widetilde{M}$ in the following three steps. Let

$$
\xi \in \Lambda \Omega(\widetilde{M})=\Omega(\widetilde{M}) \otimes \Lambda_{-1}^{\infty} \mathfrak{s l}(2, \mathbb{C})_{\sigma},
$$

$\tilde{z}_{0} \in \widetilde{M}$ and $\Phi_{0} \in \Lambda_{r} \mathrm{SL}(2, \mathbb{C})_{\sigma}$. The first step consists in solving the initial value problem

$$
d \Phi=\Phi \xi, \Phi\left(\tilde{z}_{0}\right)=\Phi_{0}
$$

to obtain a unique map $\Phi: \widetilde{M} \rightarrow \Lambda_{r} \mathrm{SL}(2, \mathbb{C})_{\sigma}$. Secondly, Iwasawa decompose $\Phi=F B$ pointwise on $\widetilde{M}$ to obtain a unique map $F: \widetilde{M} \rightarrow \Lambda_{r} \mathrm{SU}(2)_{\sigma}$.

Let $\partial_{\lambda}=\frac{\partial}{\partial \lambda}$. The final step is performed by plugging $F$ into the Sym-Bobenko formula

$$
f_{\lambda}=-\frac{1}{H}\left(i \lambda\left(\partial_{\lambda} F\right) F^{-1}+\frac{i}{2} F \sigma F^{-1}\right)
$$


to obtain conformal immersions $f_{\lambda}: \widetilde{M} \rightarrow \Lambda \mathfrak{s u}(2)_{\sigma}$ with constant mean curvature $H \neq 0$; that is, for each $\lambda_{0} \in S^{1}$ we have a conformal CMC immersion $f_{\lambda_{0}}$ : $\widetilde{M} \rightarrow \mathfrak{s u}(2) \cong \mathbb{R}^{3}$. We call $\xi \in \Lambda \Omega(\widetilde{M})$ the holomorphic potential. We thus have a map $\left(\xi, \Phi_{0}, \tilde{z}_{0}\right) \mapsto f_{\lambda}$, and the triple $\left(\xi, \Phi_{0}, \tilde{z}_{0}\right)$ is called the DPW data of a CMC immersion. The metric and Hopf differential are determined by a triple $\left(\xi, \Phi_{0}, \tilde{z}_{0}\right)$ as follows. Locally, on $U \subset M$, if we write $\xi=\lambda^{-1}$ off $\left[a_{1}, a_{2}\right]+\ldots$ and $B(\lambda=0)=\operatorname{diag}\left[r, r^{-1}\right]$ for holomorphic 1 -forms $a_{i} \in \Omega(U)$ and $r: U \rightarrow \mathbb{R}^{+}$, then it can be shown that $f_{1}$ has metric $4 r^{4}\left|a_{1}\right|^{2}$ and Hopf differential $Q=-\frac{1}{2} a_{1} a_{2}$. It follows that $f_{1}$ has branch points at the zeroes of $a_{1}$ and umbilics at the zeroes of $a_{2}$. Our basis of $\mathfrak{s u}(2)$ will be

$$
e_{1}=\left(\begin{array}{cc}
i & 0 \\
0 & -i
\end{array}\right), e_{2}=\left(\begin{array}{cc}
0 & 1 \\
-1 & 0
\end{array}\right), e_{3}=\left(\begin{array}{ll}
0 & i \\
i & 0
\end{array}\right)
$$

As a consequence of using twisted loop groups in the construction, there is some redundancy in the associated family in that every surface appears twice up to rigid motion.

Lemma 3.1. Under the map $\lambda \mapsto-\lambda$ the corresponding surface is rotated about $e_{1}$ by $\pi$ radians; that is, $f_{\lambda} \mapsto e_{1} f_{\lambda} e_{1}^{-1}$.

Proof. Twistedness is $\xi(-\lambda)=\boldsymbol{\sigma} \xi(\lambda)=e_{1} \xi(\lambda) e_{1}^{-1}$. If $\Phi$ solves (3.1) with triple $\left(\xi, \Phi_{0}, \tilde{z}_{0}\right)$, then $\Phi e_{1}$ solves (3.1) with triple $\left(e_{1} \xi e_{1}^{-1}, \Phi_{0} e_{1}, \tilde{z}_{0}\right)$.

It is well known that to every CMC surface there exist two parallel surfaces, one of constant Gaussian curvature, the other of constant mean curvature, obtained by moving every point on the surface in the normal direction by $\frac{1}{2 H}$ and $\frac{1}{H}$ respectively. The parallel associated family of CMC immersions is given by

$$
f_{\lambda}^{\text {par }}=-\frac{1}{H}\left(i \lambda\left(\partial_{\lambda} F\right) F^{-1}-\frac{i}{2} F \sigma F^{-1}\right) .
$$

Lemma 3.2. Let $f_{\lambda}$ be the associated family of CMC immersions obtained from $\left(\xi, \Phi_{0}, \tilde{z}_{0}\right)$. Then the triple $\left(e_{3} \xi e_{3}^{-1}, e_{3} \Phi_{0} e_{3}^{-1}, \tilde{z}_{0}\right)$ generates the parallel associated family $f_{\lambda}^{\text {par }}$.

Proof. If $\Phi$ solves (3.1) for the triple $\left(\xi, \Phi_{0}, \tilde{z}_{0}\right)$, then $\Psi=e_{3} \Phi e_{3}^{-1}$ solves (3.1) for the triple $\left(e_{3} \xi e_{3}^{-1}, e_{3} \Phi_{0} e_{3}^{-1}, \tilde{z}_{0}\right)$ with resulting associated family $g_{\lambda}$. If $\Phi=F B$ is the Iwasawa decomposition of $\Phi$, then $\Psi=e_{3} F B e_{3}^{-1}$. Since $b_{0}=\left.B\right|_{\lambda=0}$ is diagonal, we can write $b_{0} e_{3}^{-1}=e_{3}^{-1} b_{0}^{-1}$ and thus $B e_{3}^{-1}=e_{3}^{-1} \widetilde{B}$ for some $\widetilde{B} \in \Lambda_{r}^{+} \operatorname{SL}(2, \mathbb{C})_{\sigma}$. Hence $\Psi=e_{3} F \widetilde{B} e_{3}^{-1}$, and the Sym-Bobenko formula gives

$$
\begin{aligned}
g_{\lambda} & =-\frac{1}{H}\left(i \lambda e_{3}\left(\partial_{\lambda} F\right) F^{-1} e_{3}^{-1}+\frac{1}{2} e_{3} F e_{3}^{-1} e_{1} e_{3} F^{-1} e_{3}^{-1}\right) \\
& =-\frac{1}{H} e_{3}\left(i \lambda\left(\partial_{\lambda} F\right) F^{-1}-\frac{1}{2} F e_{1} F^{-1}\right) e_{3}^{-1}=e_{3} f_{\lambda}^{p a r} e_{3}^{-1} .
\end{aligned}
$$

Therefore the surface generated by the potential $e_{3} \xi e_{3}^{-1}$ is the parallel surface rotated about $e_{3}$ by $\pi$ radians.

The left action by $\Lambda_{r} \mathrm{SU}(2)_{\sigma}$ on the initial condition, $\Phi_{0} \mapsto C \Phi_{0}$ for $C \in$ $\Lambda_{r} \mathrm{SU}(2)_{\sigma}$, results in a Euclidean motion of the surface. In fact, we have the following.

Lemma 3.3. Let $\Phi$ solve (3.1) with triple $\left(\xi, \Phi_{0}, \tilde{z}_{0}\right)$, and let $f_{\lambda}$ denote the resulting associated family. Let $C \in \Lambda_{r} \mathrm{SU}(2)_{\sigma}$ and for $\alpha \in \mathbb{C}$ with $|\alpha|=1$ define $A=$ 
$\operatorname{diag}[\alpha, \bar{\alpha}]$. Then $\Psi=C \Phi A$ solves (3.1) for the triple $\left(A^{-1} \xi A, C \Phi_{0} A, \tilde{z}_{0}\right)$, and the resulting associated family $g_{\lambda}$ is given by

$$
g_{\lambda}=-\frac{1}{H} i \lambda\left(\partial_{\lambda} C\right) C^{-1}+C f_{\lambda} C^{-1} .
$$

Proof. If $\Phi=F B$ is the Iwasawa decomposition of $\Phi$, then $A^{-1} B A \in \Lambda_{r}^{+} \mathrm{SL}(2, \mathbb{C})_{\sigma}$ and the Iwasawa decomposition of $\Psi$ is $\Psi=C F B A=C F A A^{-1} B A$. Hence $\Psi$ has the unitary frame $C F A$, which inserted into the Sym-Bobenko formula gives

$$
\begin{aligned}
g_{\lambda} & =-\frac{1}{H}\left(i \lambda\left(\partial_{\lambda} C F A\right)(C F A)^{-1}+\frac{1}{2}(C F A) e_{1}(C F A)^{-1}\right) \\
& =-\frac{1}{H}\left(i \lambda\left(\left(\partial_{\lambda} C\right) C^{-1}+C\left(\partial_{\lambda} F\right) F^{-1} C^{-1}+\frac{1}{2} C F e_{1} F^{-1} C^{-1}\right)\right) \\
& =-\frac{1}{H} i \lambda\left(\partial_{\lambda} C\right) C^{-1}+C f_{\lambda} C^{-1},
\end{aligned}
$$

and proves the result.

\section{THE PERIOD PROBLEM}

Let $M$ be a connected Riemann surface with universal cover $\widetilde{M}$, and let $\Delta$ be the group of deck transformations. Let $\xi \in \Lambda \Omega(\widetilde{M})$ with $\gamma^{*} \xi=\xi$ for all $\gamma \in \Delta$. Let $\Phi: \widetilde{M} \rightarrow \Lambda_{r} \mathrm{SL}(2, \mathbb{C})_{\sigma}$ be a solution of the differential equation $d \Phi=\Phi \xi$. Writing $\gamma^{*} \Phi=\Phi \circ \gamma$ for $\gamma \in \Delta$, we define $\chi(\gamma) \in \Lambda_{r} \operatorname{SL}(2, \mathbb{C})_{\sigma}$ by $\chi(\gamma)=\left(\gamma^{*} \Phi\right) \Phi^{-1}$. The matrix $\chi(\gamma)$ is called the monodromy matrix of $\Phi$ with respect to $\gamma$.

If $\widehat{\Phi}: \widetilde{M} \rightarrow \Lambda_{r} \mathrm{SL}(2, \mathbb{C})_{\sigma}$ is another solution of $d \Phi=\Phi \xi$ and $\widehat{\chi}(\gamma)=\left(\gamma^{*} \widehat{\Phi}\right) \widehat{\Phi}^{-1}$, then there exists a constant $C \in \Lambda_{r} \operatorname{SL}(2, \mathbb{C})_{\sigma}$ such that $\widehat{\Phi}=C \Phi$. Hence $\widehat{\chi}(\gamma)=$ $C \chi(\gamma) C^{-1}$ and different solutions give rise to mutually conjugate monodromy matrices.

A choice of base point $\tilde{z}_{0} \in \widetilde{M}$ and initial condition $\Phi_{0} \in \Lambda_{r} \mathrm{SL}(2, \mathbb{C})_{\sigma}$ gives the monodromy representation $\chi: \Delta \rightarrow \Lambda_{r} \mathrm{SL}(2, \mathbb{C})_{\sigma}$ of $\xi$. Henceforth, when we speak of the monodromy representation, or simply monodromy, we tacitly assume that it is induced by an underlying triple $\left(\xi, \Phi_{0}, \tilde{z}_{0}\right)$ with invariant holomorphic potential $\gamma^{*} \xi=\xi$ for all $\gamma \in \Delta$. It is shown in [2] that CMC immersions of open Riemann surfaces $M$ can always be generated by such invariant holomorphic potentials.

If $\Phi=F B$ is the pointwise Iwasawa decomposition of $\Phi: \widetilde{M} \rightarrow \Lambda_{r} \operatorname{SL}(2, \mathbb{C})_{\sigma}$, then we shall need to study the monodromy of $F$ as a means to controlling the periodicity of the resulting immersion (3.2). A priori, we are not assured that the quantity $\mathcal{M}(\gamma)=\left(\gamma^{*} F\right) F^{-1}$ is $z$-independent for all $\gamma \in \Delta$. One way to circumvent this issue is to ensure that $\chi$ is $\Lambda_{r} \mathrm{SU}(2)_{\sigma}$-valued.

Lemma 4.1. Let $\chi(\tau)$ be the monodromy of $\xi$ for $\tau \in \Delta$. If $\chi(\tau) \in \Lambda_{r} \mathrm{SU}(2)_{\sigma}$, then $\chi(\tau)=\mathcal{M}(\tau)$.

Proof. From the Iwasawa decomposition $\Phi=F B$ we obtain $\chi(\tau) F B=\tau^{*} F \tau^{*} B$. If $\chi(\tau) \in \Lambda_{r} \mathrm{SU}(2)_{\sigma}$, then (2.1) yields $\left(\tau^{*} F\right)^{-1} \chi(\tau) F=\left(\tau^{*} B\right) B^{-1}=$ Id. Therefore $\chi(\tau)=\mathcal{M}(\tau)$.

The condition $\chi(\tau) \in \Lambda_{r} \mathrm{SU}(2)_{\sigma}$ can be ensured if the initial condition $\Phi_{0}$ in (3.1) is unitary and the potential $\xi$ is skew Hermitian along a curve $\tau \in \pi_{1}(M)$ that passes through the base point.

Lemma 4.2. Let $\xi \in \Lambda \Omega(\widetilde{M})$ and $\tau^{*} \xi=\xi$ for $\tau \in \pi_{1}(M) \cong \Delta$. Pick a point $z_{0} \in \tau$ and $\Phi_{0} \in \Lambda_{r} \mathrm{SU}(2)_{\sigma}$, and let $\Phi: \widetilde{M} \rightarrow \Lambda_{r} \mathrm{SL}(2, \mathbb{C})_{\sigma}$ be the solution of (3.1) with triple $\left(\xi, \Phi_{0}, z_{0}\right)$. If $\xi$ is $\Lambda \mathfrak{s u}(2)_{\sigma}$-valued along $\tau$, then $\chi(\tau) \in \Lambda_{r} \mathrm{SU}(2)_{\sigma}$. 


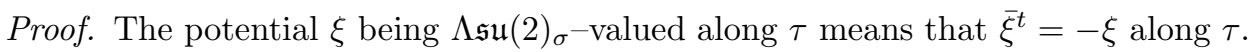
Hence for $\xi=\Phi^{-1} d \Phi$ we have $d \Phi \bar{\Phi}^{t}+\Phi d \bar{\Phi}^{t}=0$. Integrating this last equation gives $\Phi \bar{\Phi}^{t}=C$ for a $z$-independent $C \in \Lambda_{r} \mathrm{SL}(2, \mathbb{C})_{\sigma}$. The initial condition satisfies $\Phi_{0}{\overline{\Phi_{0}}}^{t}=\mathrm{Id}$ forcing $C=\mathrm{Id}$. Hence $\left.\Phi\right|_{z \in \tau} \in \Lambda_{r} \mathrm{SU}(2)_{\sigma}$, ensuring that $\chi(\tau) \in$ $\Lambda_{r} \mathrm{SU}(2)_{\sigma}$.

The next result characterises the period problem in the DPW framework.

Theorem 4.3 ([3]). Let $\xi \in \Lambda \Omega(\widetilde{M})$ have $\Lambda_{r} \mathrm{SU}(2)_{\sigma}$-valued monodromy $\chi$, and let $f_{\lambda}$ be the associated family generated by the triple $\left(\xi, \Phi_{0}, \tilde{z}_{0}\right)$. Then there exists a $\lambda_{0} \in S^{1}$ such that $\tau^{*} f_{\lambda_{0}}=f_{\lambda_{0}}$ for $\tau \in \Delta$ if and only if $\chi(\tau, \lambda)$ satisfies both

$$
\begin{aligned}
\chi\left(\tau, \lambda_{0}\right) & = \pm \mathrm{Id}, \\
\left.\partial_{\lambda} \chi(\tau)\right|_{\lambda_{0}} & =0 .
\end{aligned}
$$

\section{Delaunay surfaces}

As an application of the above, we shall construct the holomorphic data in the DPW representation that generates the family of Delaunay surfaces. The conformal structure of a Delaunay surface is that of a twice punctured Riemann sphere. So we let $M=\mathbb{C}^{*}$. If we identify $\mathbb{C}^{*} \cong \mathbb{C} / 2 \pi i \mathbb{Z}$, then exp : $\mathbb{C} \rightarrow \mathbb{C}^{*}$ is the universal covering map and we shall write $\tilde{z}=\log (z)$. The group of deck transformations $\Delta \cong \mathbb{Z}$ is generated by $\tau: \tilde{z} \mapsto \tilde{z}+2 \pi i$. In the associated family, we shall solve the period problem (4.1) and (4.2) for $\lambda_{0}=1$, and choose the base point $\tilde{z}_{0}=0$. Consider

$$
\xi=\xi_{-1} \frac{d z}{z} \text { with } \xi_{-1}=\left(\begin{array}{cc}
c & a \lambda^{-1}+\bar{b} \lambda \\
b \lambda^{-1}+\bar{a} \lambda & -c
\end{array}\right)
$$

with $a, b \in \mathbb{C}$ and $c \in \mathbb{R}$. The two eigenvalues of $\xi_{-1}$ are $\pm \sqrt{-\operatorname{det} \xi_{-1}}$. Since the residue is invariant under $z \mapsto \alpha z$ for $|\alpha|=1$, the induced surface has intrinsic rotational symmetry. The solution to (3.1) with triple $(\xi, \mathrm{Id}, 0)$ is obtained by exponentiation and with $z=r e^{i t}$ can be factored as

$$
\Phi(z, \lambda)=\exp \left(\log (z) \xi_{-1}\right)=\exp \left(i t \xi_{-1}\right) \exp \left(\ln |r| \xi_{-1}\right) .
$$

Lemma 5.1. $F_{1}(t):=\exp \left(i t \xi_{-1}\right) \in \Lambda \mathrm{SU}(2)_{\sigma}$ is a one-parameter family of rotations with common axis $i \xi_{-1}$.

Proof. Since $\xi_{-1}$ is Hermitian, $i t \xi_{-1} \in \mathfrak{s u}_{2}$ for all $t \in \mathbb{R}$. Hence $F_{1}(t) \in \Lambda \mathrm{SU}(2)_{\sigma}$ and $\left[i \xi_{-1}, F_{1}(t)\right]=0$. The claim now follows by uniqueness of the axis.

The differential equation for a profile curve $r(t)$ of a CMC $H$ surface of revolution is given by

$$
\frac{\ddot{r}+1 / r}{\left(1+\dot{r}^{2}\right)^{3 / 2}}=2 H
$$

which is equivalent to the first-order equation $\dot{s}=0$ for $s(t)=\frac{r}{\sqrt{1+\dot{r}^{2}}}-r^{2} H$. At a neck or a bulge we have $\dot{r}=0$. So there is a constant $\kappa$ such that $r(1-r H)=\kappa$. In 1841 Delaunay [1] proved the following theorem. An elementary proof is given by Smyth [14.

Theorem 5.2 ([1]). A surface of revolution is a constant mean curvature surface if and only if the profile curve is a roulette of a conic. 
The resulting one-parameter family of CMC cylinders, complete immersed surfaces of revolution, are called Delaunay surfaces. A Delaunay surface is uniquely determined by the bulge and neck radii up to rigid motions. The surfaces obtained from ellipses are called unduloidal, and the surfaces obtained from parabolas are called nodoidal [5. In particular, Delaunay surfaces have no umbilic point. A quadratic meromorphic differential on $\mathbb{C P}^{1}$ has degree -4 . For an unbranched cylinder without umbilics, the four poles of the Hopf differential have to be at the ends. By translational symmetry, the Hopf differential of a Delaunay surface has to have a pole of order 2 at each end. Hence, up to coordinate transformations, the Hopf differential for a Delaunay surface is of the form $z^{-2} d z^{2}$.

Theorem 5.3. Let $\xi=\xi_{-1} \frac{d z}{z}$ with $\xi_{-1}$ as in (5.1). If $|a+\bar{b}|^{2}+c^{2}=1 / 4$ and $a b \in \mathbb{R}$, then the triple $(\xi, \mathrm{Id}, 0)$ generates the associated family of Delaunay surfaces which at $\lambda=1$ has bulge/neck radius

$$
\frac{1}{2 H}(1 \pm \sqrt{1-16 a b}) .
$$

Proof. Diagonalising $\xi_{-1}$, the solution to (3.1) with triple $(\xi, \mathrm{Id}, 0)$ is given by

$$
\Phi(z, \lambda)=T(\lambda) \operatorname{diag}\left[z^{-\mu(\lambda)}, z^{\mu(\lambda)}\right] T(\lambda)^{-1} \text { where } T(\lambda)=\left(\begin{array}{cc}
\frac{(c-\mu) \lambda}{\bar{a} \lambda^{2}+b} & \frac{(c+\mu) \lambda}{\bar{a} \lambda^{2}+b} \\
1 & 1
\end{array}\right)
$$

and $\mu(\lambda)=\sqrt{a \bar{a}+b \bar{b}+c^{2}+a b \lambda^{-2}+\overline{a b} \lambda^{2}}$. Since $\xi \in \Lambda^{\sigma} \mathfrak{s u}_{2}$ along $|z|=1$, the monodromy is $\Lambda_{r} \mathrm{SU}(2)_{\sigma}$-valued and given by

$$
\chi(\lambda)=T(\lambda) \operatorname{diag}\left[e^{-2 \pi i \mu(\lambda)}, e^{2 \pi i \mu(\lambda)}\right] T(\lambda)^{-1} .
$$

The first closing condition $\chi(1)= \pm \mathrm{Id}$ is equivalent to $\mu(1) \in \frac{1}{2} \mathbb{Z}$. For the constants $a, b, c$ this holds if and only if $|a+\bar{b}|^{2}+c^{2} \in \frac{1}{4} \mathbb{Z}$. The second closing condition $\left.\partial_{\lambda} H\right|_{\lambda=1}=0$ holds if $\left.\partial_{\lambda} \mu\right|_{\lambda=1}=0$, which is equivalent to $a b \in \mathbb{R}$ if the first closing condition is assumed. Let arg denote some fixed branch of argument. Then since $a b \in \mathbb{R}$, we have $\arg (\bar{b})=\arg (a)$. Let $\gamma=e^{i \arg (a)}$, and define $A=\operatorname{diag}[\sqrt{\gamma}, \sqrt{\bar{\gamma}}]$. By Lemma 3.3 the surface generated by the triple $\left(A \xi A^{-1}, \mathrm{Id}, 1\right)$ is a rotation of the surface generated by the triple $(\xi, \mathrm{Id}, 1)$, but since the coefficients of $\lambda^{-1}, \lambda$ in the potential $A \xi A^{-1}$ are real, we can assume without loss of generality that $a, b \in \mathbb{R}$ and the two closing conditions together with the requirement that the surface be simply wrapped, reduce to the equation of an elliptic cylinder $(a+b)^{2}+c^{2}=\frac{1}{4}$. This leaves two real parameters of freedom, the neck size of the surface and the radius of the image circle of $S^{1}$. At the base point $z_{0}=1$ we have $\Phi(1, \lambda)=\mathrm{Id}$, and the image under the immersion is $f(1, \lambda)=-\frac{1}{2 H} e_{1}$. By Lemma 5.1 the frame along $|z|=1$ is given by the one-parameter family of $\operatorname{rotations} \exp \left(i t \xi_{-1}\right)$ with common axis $i \xi_{-1}$. Hence the resulting surface is a surface of revolution and thus a Delaunay surface. The unit circle $|z|=1$ is mapped to the circle with centre

$$
\frac{1}{2}(f(1, \lambda)-f(-1, \lambda))=\frac{8 a^{2}+8 a b-1}{2 H} e_{1}-\frac{4 a c}{H} e_{3},
$$

radius $2\left|a H^{-1}\right|$ and axial direction $i \xi_{-1}$. The angle $\theta$ between the axis and the tangent vector of the profile curve $r(t)$ at some $r_{0}$ is given by

$$
\cos \theta=\frac{1}{\sqrt{1+\dot{r}^{2}}}=2(a+b) \text {. }
$$


The quadratic equation at a neck or bulge is

$$
r(1-r H)=2(a+b) 2|a / H|-4 H|a / H|^{2},
$$

whose solutions are given by (5.4).

By Lemma 3.2, the parallel CMC surface of a Delaunay surface is obtained by interchanging neck and bulge. We conclude our discussion of Delaunay surfaces by indicating how the choice of the constants determines the resulting surface. The case $a b>0$ results in unduloids, while $a b<0$ yields nodoids. For $a=b=1 / 4$, the resulting surface is the round cylinder and the limiting case $a=1 / 2, b=0$ produces a round sphere with two points removed.

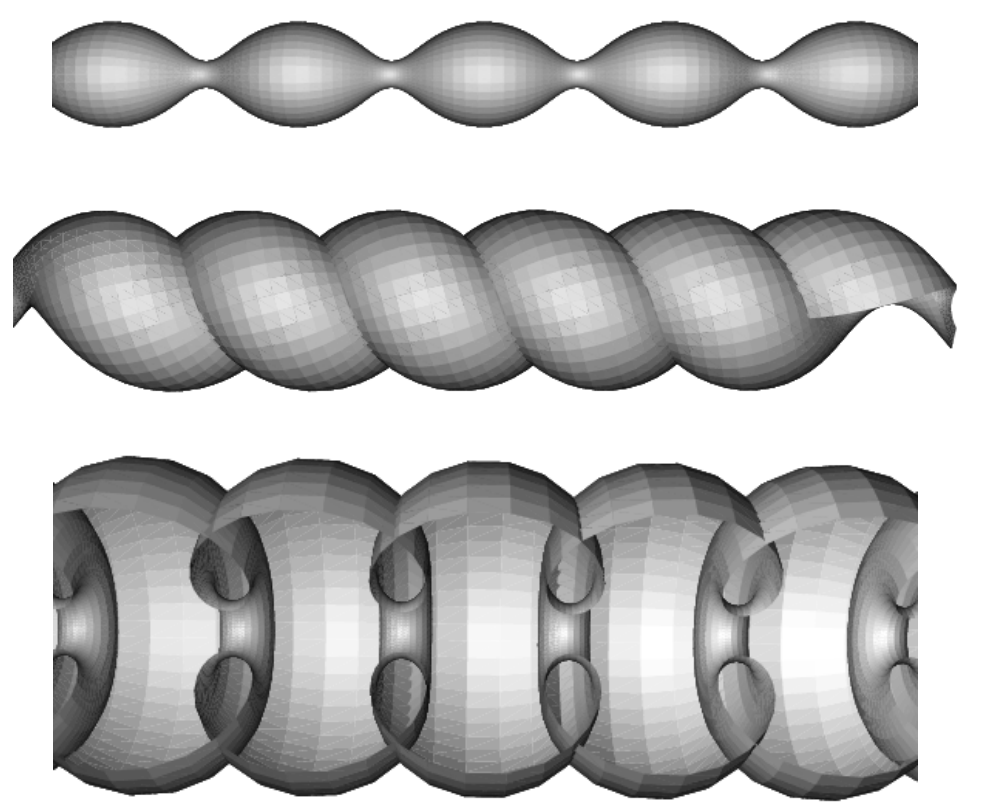

Figure 1. An unduloid $\left(a=.4, b=.1, \lambda_{0}=1\right)$, an intermediate member in the associated family of an unduloid $(a=.4, b=$ $.1, \lambda_{0}=\exp (2 \pi i \theta)$ with $\left.\theta=.25882\right)$ and a cutaway view of a nodoid $\left(a=.6, b=-.1, \lambda_{0}=1\right)$. These images were produced using software by N. Schmitt [12].

\section{REFERENCES}

1. C. Delaunay, Sur la surface de révolution dont la courbure moyenne est constante, J. Math. Pures et Appl. Sér. 16 (1841), 309-320.

2. J. Dorfmeister and G. Haak, Construction of non-simply connected CMC surfaces via dressing, J. Math. Soc. Japan, 55 (2003), no. 2, 335-364. MR 2004d:53010

3. (1998), 229-287. MR 99f:53007

4. J. Dorfmeister, F. Pedit, and H. Wu, Weierstrass type representation of harmonic maps into symmetric spaces, Comm. Anal. Geom. 6 (1998), no. 4, 633-668. MR 2000d:53099

5. J. Eells, The surfaces of Delaunay, Math. Intelligencer 9 (1987), 53-57. MR 88h:53011 
6. M. Kilian, Constant mean curvature cylinders, Ph.D. thesis, Univ. of Massachusetts, Amherst, 2000 .

7. M. Kilian, I. McIntosh, and N. Schmitt, New constant mean curvature surfaces, Experiment. Math. 9 (2000), no. 4, 595-611. MR 2002e:53008

8. M. Kilian, N. Schmitt, and I. Sterling, Dressing CMC n-Noids, Math. Z. 246 (2004), no. 3, 501-519.

9. N. Korevar, R. Kusner, and B. Solomon, The structure of complete embedded surfaces with constant mean curvature, J. Diff. Geom. 30 (1989), no. 2, 465-503. MR 90g:53011

10. I. McIntosh, Global solutions of the elliptic 2d periodic Toda lattice, Nonlinearity 7 (1994), no. 1, 85-108. MR 95g:58108

11. E. A. Ruh and J. Vilms, The tension field of the Gauss map, Trans. Amer. Math. Soc. 149 (1970), 569-573. MR 41:4400

12. N. Schmitt, CMCLab, http://www.gang.umass.edu/software.

13. _ Constant mean curvature trinoids, arXiv:math.DG/0403036.

14. B. Smyth, A generalization of a theorem of Delaunay on constant mean curvature surfaces, IMA Vol. Math. Appl. 51 (1993), 123-130. MR 94f:53012

15. K. Uhlenbeck, Harmonic maps into lie groups (classical solutions of the chiral model), J. Diff. Geom. 30 (1989), 1-50. MR 90g:58028

Department of Mathematical Sciences, University of Bath, Bath, BA2 7AY, United KINGDOM

E-mail address: masmk@maths.bath.ac.uk 